\title{
Politisch informierte und äußerst informative Gesetzgebungslehre: Kunst des Möglichen anstatt „Gesetzgebungskultur“
}

\section{Fliedner, Ortlieb: Rechtsetzung in Deutschland. Gesetzgebung in der Demokratie, Nomos Ver- lagsgesellschaft, Baden-Baden 2013, 153 Seiten, € 39,-.}

Das Buch von Rechtsanwalt Ortlieb Fliedner, der auf frühere Erfahrungen als Ministerialbeamter und als Mitarbeiter einer Bundestagsfraktion zurückgreifen kann, will Grundfragen, politische Bedingungen und Methoden der Gesetzgebung einer Klientel nahebringen, von der gemeinhin angenommen wird, sie wisse darüber Bescheid: nämlich den Juristen. Das ist nicht etwa paradox; der Autor weiß, dass dem nicht so ist. Die Juristenausbildung in Deutschland ist seit jeher auf die Anwendung des Rechts, nicht auf seine Erzeugung fokussiert, und daran hat sich ein Jahrhundert lang nichts Entscheidendes geändert. Dass Fliedner einführend feststellen muss, es gebe in Deutschland nach wie vor an keiner Universität einen Lehrstuhl für Gesetzgebung - neben „Heerscharen“ von Juristen, die sich mit der Auslegung des geltenden Rechts befassen (S. 11) -, belegt nach seiner Auffassung nichts geringeres als eine parlamentarisch-demokratische Sehstörung in der Juristenausbildung.

Das wird sogleich klar in dem kurzen Rückblick auf die seit den Zeiten Otto von Bismarcks kaum gewandelten Vorstellungen über Recht, Politik und Gesetz. Der Autor drückt sich hier noch relativ höflich aus, wenn er die Defizite auf gängige Meinungen der Sorte zurückführt, Politik sei eben ein „schmutziges Geschäft“, sie stehe im Gegensatz zu gerechten Gesetzen, und sie sei schuld an zu vielen und zu schlechten Gesetzen oder verhindere das Zustandekommen guten Rechts. Die eigentliche Frage ist, woher diese gängigen Auffassungen kommen, wodurch sie aufrecht erhalten werden und warum nach nunmehr 65 Jahren Grundgesetz noch immer erläutert werden muss, dass „Recht in der Demokratie ausschließlich Menschenwerk“ ist (S. 15). Solange das nicht wirklich begriffen wird, solange nach dem „guten“ oder „richtigen“ Gesetz als solchem gefahndet wird, so lange wird auch der Erkenntnis ausgewichen, dass in die Gesetzgebung all die Unsicherheit, Vorläufigkeit, Widersprüchlichkeit und Unstimmigkeit mit eingehen muss, die das Wesen der Politik in der Demokratie ausmacht, denn: „Gesetze sind ... das Ergebnis politischer Diskussionen und Entscheidungen ... Recht ist in der Demokratie durch Gesetze ... verbindlich gewordene Politik“ (S. 17).

Die in „Kapitel 1 Recht in der Demokratie“ (S. 13 ff.) behandelten Grundfragen, darunter der „tiefe Graben zwischen Recht und Politik“ (S. 18) nebst Zitaten von höchst fragwürdigen diesbezüglichen Äußerungen verschiedener Rechtsgelehrter, bilden einen besonders lesenswerten Abschnitt dieser Schrift. Sie ist indessen keine „Streitschrift“ in diesem Sinne, und sie ist nicht - will wohl auch nicht sein - eine tiefgehende wissenschaftliche Untersuchung dieser Probleme. Fliedner geht es eher darum, auf dieser Grundlage eine bessere, also politisch und verfassungspolitisch besser informierte Gesetzgebungslehre vorzulegen als diejenigen, die in der Fachpublizistik vorzufinden sind (über sie gibt das Literaturverzeichnis S. $149 \mathrm{ff}$. Auskunft, allerdings etwas lückenhaft). Dem dienen die folgenden Abschnitte über „Ansätze zu einer besseren Gesetzgebung“ (S. 37 ff.), „Gestaltungsaspekte guter Gesetze“ (S. 55 ff.), „Das Gesetzgebungsverfahren“ (S. 89 ff.) und „Weitere Aspekte der Rechtsetzung“ (S. 133 ff.).

In diesen Abschnitten findet sich viel Wissenswertes, besonders durch die Berücksichtigung möglichst aller Institutionen und Akteure sowie der von ihnen anzuwendenden Pro- 
zeduren, die andernorts oft weniger vollständig oder mit unzureichender Gewichtung berücksichtigt sind. In der Darstellung der zahlreichen Beteiligten mit ihren Rollen bei der Herstellung des geschriebenen Rechts wird sichtbar, dass es „den“ Gesetzgeber, der die Gesetze „gibt“, nicht gibt. Es handelt sich in der Wirklichkeit um einen hochkomplexen Prozess politischer und fachlicher Initiativ-, Partizipations- und Vetopositionen, in dem Beiträge unterschiedlichster, auch kontroverser Art verarbeitet werden und der von keiner Stelle aus durchgehend gesteuert werden kann. Mit seiner bei aller Knappheit doch umfassenden Einbeziehung der zahlreichen Mitwirkenden mit ihren je eigenen Interessen und Logiken gelingt Fliedner ein überzeugendes Plädoyer dafür, dass Juristen diesen Prozess besser verstehen und in Rechnung stellen sollten, wenn sie Gesetze anwenden, auslegen und beurteilen oder an ihrer Erzeugung mitwirken.

Jedoch wird die Darstellung mit dem Eindringen in die praktischen Details formaler und herkömmlicher. Es gelingt dem Autor nur teilweise, den kritisch-systematischen Anspruch der Einführung durch die gesamte Darstellung durchzuhalten. Das ist vor allem dem Bemühen geschuldet, die zahlreichen - auch von politischer Seite regelmäßig dargebotenen - Konzepte für eine bessere, rationalere, widerspruchsfreiere, wirkungsvollere etc. Gesetzgebung einigermaßen vollständig zu referieren. Dadurch geraten die an vielen Stellen beigefügten kritischen Erwägungen zwar zu durchaus anschlussfähigen Kommentaren, ohne dass aber die diesbezügliche Praxis grundsätzlich in Frage gestellt wird.

So ist es zum Beispiel nur zu berechtigt, die routinemäßige Klage über eine angebliche Gesetzesflut als eine „Mär“ zu kennzeichnen (S. 46) und sowohl die Zahlen als solche richtigzustellen als auch die Aussagekraft solcher Zahlen zu hinterfragen. Ebenso zutreffend ist die Einschätzung, dass die oft geforderte „Notwendigkeitsprüfung“ von Gesetzen kein sinnvoller Ansatz für gesetzestechnische Verbesserungen, sondern eine politische Willensentscheidung ist (S. 49). Aber was folgt aus diesen und zahlreichen weiteren kritischen Anmerkungen für die Forderung nach „Verbesserung“ überhaupt? Wer kann angesichts der genuin politischen Natur der Normsetzung für die Gesellschaft legitimiert und befähigt sein, die Ergebnisse zu verbessern? Wenn die meisten herkömmlichen Ansätze, ob auf nationaler oder auf EU- und OECD-Ebene (dazu S. 43 ff.), mehr oder weniger auf interessenimprägnierte Sonntagsreden und politische Willfährigkeit gegenüber publizistischen Unmutsäußerungen zurückzuführen sind, fragt sich doch, inwieweit sie weiter verfolgt werden sollten. Nach der Einführung in das Buch wäre ein Votum in der Richtung vorstellbar, dass es eben nur das gewählte Parlament allein sein kann, das in seinem Willensbildungsprozess das Ergebnis dessen herbeiführt und dann auch politisch zu verantworten hat, was wir „Gesetzgebung“ nennen - das Ergebnis einschließlich der vermeintlichen „Fehler“ und „Unzulänglichkeiten“, die den politischen Prozess der Repräsentation einer pluralistischen Gesellschaft widerspiegeln - und dies gerade auch in Sprache, Struktur und Systematik des geschriebenen Rechts. Das von Fliedner angemahnte Desiderat besteht ja gerade darin, dass die anwendungsorientierten Juristen die politisch-demokratische Genetik des gesetzten Rechts zu akzeptieren haben, statt es in der Auslegung davon säubern zu wollen.

Wohin die Forderungen nach „optimaler Methodik“ in der Gesetzgebung führen, lässt sich in der Rechtsprechung des Bundesverfassungsgerichts besichtigen: zur Delegitimierung der politisch-repräsentativen Willensbildung durch Vorgaben von „Sachgerechtheit“, die ihrerseits weder politisch legitimiert noch inhaltlich hinterfragt, wohl aber politisch äußerst wirkungsvoll sind (oft genug sind sie von organisierten Interessen entwickelt). Dagegen hilft der von Fliedner letztlich akzeptierte Ansatz - der mehr oder weniger allen Optimierungs- 
konzepten zugrunde liegt - nicht wirklich weiter, demzufolge Qualitätskriterien oder Standards gefunden werden müssten, die „unabhängig von einer politischen Bewertung des Gesetzesinhalts gelten“ können und die ,jeder Gesetzgeber ... berücksichtigen sollte“ (S. 60). Genau in dieser gedachten oder gewünschten Unterscheidung liegt das Problem: Solche Standards können nur entweder trivial sein, oder sie richten sich gegen das Politisch-Repräsentative in der Normsetzung. Übrigens sind viele „nur“ gesetzestechnischen Verbesserungsmöglichkeiten keineswegs trivial. Der Jurist weiß, wie ein Satzzeichen, ein Absatz oder eine Reihung in einem Gesetzestext die Interpretationsmöglichkeiten verändern können.

Einwände wie diese richten sich nicht zuerst gegen das hier besprochene Buch, sondern generell gegen die Erwartungen an eine wie auch immer konzipierte „Kunst“ (oder, noch schöner und modischer, eine „Kultur“) der Gesetzgebung, mit der „bessere“ Gesetze möglich werden sollen. Es gibt sie nicht; was bleibt, ist allenfalls die „Kunst des Möglichen“, wie sie der Politik insgesamt zugeschrieben wird und die am Ende auch für die Normerzeugung gelten kann. Das Buch von Fliedner ist ungeachtet des hier skizzierten Grundproblems äußerst informativ und sollte allseits herangezogen werden: von Juristen, um sich der Fragwürdigkeit einer nur scheinbar unpolitischen Beschränkung auf die Auslegung und Anwendung des geschriebenen Rechts bewusst zu werden; und von Politikwissenschaftlern, um an der Auffüllung des vom Autor konstatierten Grabens zwischen Recht und Politik mitwirken zu können.

Wolfgang Zeh

\section{Gesetzgebung: Entstehung und Wandel aus theoretischer und praxisorientierter Sicht}

Fleischer, Holger (Hrsg.): Mysterium „Gesetzesmaterialien“. Bedeutung und Gestaltung der Gesetzesbegründung in Vergangenheit, Gegenwart und Zukunft, Mohr Siebeck, Tübingen 2013, 135 Seiten, € 34,-.

Kluth, Winfried und Günter Krings (Hrsg.) in Verbindung mit der Deutschen Gesellschaft für Gesetzgebung: Gesetzgebung: Rechtsetzung durch Parlamente und Verwaltungen sowie ihre gerichtliche Kontrolle, C.F. Müller, Heidelberg 2014, 1050 Seiten, € 199,--

Wer ein Gesetz lesen, verstehen, an seinem Vollzug, seiner Auslegung, Beurteilung und möglichen Fortentwicklung mitwirken will, muss sich nicht nur den Gesetzeswortlaut aneignen, sondern muss dazu auch die Gesetzesmaterialien heranziehen. Das sind in den meisten Gesetzgebungsverfahren zwei Dokumente: der Entwurf und die regelmäßig in einer Drucksache vereinte Beschlussempfehlung mit dem Bericht des federführenden Ausschusses. Beide Quellen in einer Zusammenschau mit dem Normtext und seiner amtlichen Begründung ermöglichen so den erforderlichen Erkenntnisgewinn, der zum Gesetzesvollzug und zur forensischen Anwendung unabdingbar ist.

Dass solch ein Pfad zur Norm dennoch in der Praxis nicht immer so unbeschwert funktioniert, darauf deutet der Titel der von Holger Fleischer, Direktor am Max-Planck-Institut für ausländisches und internationales Privatrecht in Hamburg, vorgelegten Aufsatzsammlung: Mysterium „Gesetzesmaterialien“ hin. Der Herausgeber und fünf weitere Autoren beleuchten und erhellen darin die Bedeutung und Gestaltung der Gesetzesbegründung in Vergangenheit, 